\title{
Renal dysfunction in a mouse model of GDM is prevented by metformin through MAPKs
}

\author{
ZHENGFEI LIU, XINYANG YU, CHAO TONG and HONGBO QI \\ Department of Obstetrics and Gynecology, The First Affiliated Hospital of Chongqing Medical University, \\ Chongqing 400016, P.R. China
}

Received June 19, 2018; Accepted February 15, 2019

DOI: $10.3892 / \mathrm{mmr} .2019 .10060$

\begin{abstract}
Gestational diabetes mellitus (GDM) incidence is increasing worldwide. In the present study, the effects of metformin on high fat diet (HFD)-induced renal dysfunction were investigated in GDM mice. In addition, the molecular mechanisms underlying metformin function were examined. GDM was induced by feeding pregnant mice a HFD, and mice were treated with two different doses of metformin (300 and $600 \mathrm{mg} / \mathrm{kg} / \mathrm{day}$ ) or PBS between embryonic day 11.5 (E11.5) and E17.5. The pregnant mice at E18.5 were utilized to analyze the effects of metformin on renal dysfunction. Renal function and the protein expression levels of inflammatory cytokines and factors of the mitogen-activated protein kinase (MAPK) signaling pathway in the kidneys of pregnant mice were assessed by ELISA and western blotting. Data obtained during late pregnancy suggested that metformin significantly decreased body weight and the levels of blood glucose in GDM mice, as assessed by the glucose tolerance test and the homeostatic model assessment for insulin resistance. The levels of microalbumin and serum $\beta 2$-microglobulin in GDM mice during late pregnancy were decreased following treatment with metformin. Furthermore, serum levels of interleukin (IL)-6 and tumor necrosis factor (TNF)- $\alpha$, and phosphorylation of MAPK1/3, MAPK14 and MAPK8 in the kidneys were decreased in GDM mice following metformin treatment at E18.5, compared with the untreated GDM group. The present study suggested that inflammation may be associated with
\end{abstract}

Correspondence to: Dr Hongbo Qi, Department of Obstetrics and Gynecology, The First Affiliated Hospital of Chongqing Medical University, 1 Youyi Road, Yuzhong, Chongqing 400016, P.R. China E-mail: qihongbocy@gmail.com

Abbreviations: GDM, gestational diabetes mellitus; T2DM, type 2 diabetes mellitus; DN, diabetic nephropathy; TNF- $\alpha$, tumor necrosis factor- $\alpha$; IL-6, interleukin-6; MAPK, mitogen-activated protein kinase; p-MAPK, phosphorylated-MAPK; ECL, enhanced chemiluminescence; OGTT, oral glucose tolerance test; HFD, high-fat diet; LFD, low-fat diet; STZ, streptozotocin

Key words: metformin, GDM, renal dysfunction, inflammation, MAPKs renal dysfunction in GDM mice, and that the MAPK signaling pathway may be involved in the protective effect of metformin on renal dysfunction in GDM mice.

\section{Introduction}

Gestational diabetes mellitus (GDM) incidence is increasing worldwide, and represents a severe medical problem. In total, $\sim 18 \%$ of pregnant women are diagnosed with GDM according to the guidelines of The International Association of Diabetes and Pregnancy Study Groups (1). GDM may cause adverse outcomes in the mother and newborn during pregnancy and following birth (2-4). The risk of women with GDM to develop type 2 diabetes mellitus (T2DM) following delivery has been reported to increase by 7 times when compared with healthy women (5). The mechanism of GDM involves the presence of dysfunctional pancreatic $\beta$-cells that are not able to secrete high levels of insulin and to compensate for insulin resistance in late pregnancy. In GDM, the function of $\beta$-cells declines and glucose intolerance may be detected in the mother in the first year following delivery $(6,7)$. Therefore, the symptoms of GDM may be used as predictive factors for the development of prediabetes and T2DM, and these two conditions share similar pathophysiological and genetic characteristics (8).

Patients with T2DM present a $40 \%$ risk of developing diabetic nephropathy (DN), which has been the leading cause of end-stage renal failure worldwide (9). DN was identified by previous studies to be associated with mitogen-activated protein kinases (MAPKs) (10) and with the signaling pathways involved in the pro-inflammatory response (11). The pro-inflammatory response may cause the release of inflammatory cytokines, including tumor necrosis factor- $\alpha$ (TNF- $\alpha$ ) and interleukin-6 (IL-6), leading to kidney damage in patients with diabetes (11). A number of previous studies demonstrated that GDM exhibits a long-term effect on renal function following delivery $(12,13)$. Beharier et al $(12)$ observed that GDM represents a significant risk factor for long-term renal morbidity, and identified a linear correlation between the number of GDM episodes and the risk of developing renal morbidity. Metformin is a standard clinical drug used to treat T2DM and GDM, and its safety and efficacy were demonstrated in a number of previous studies (14-16). However, the mechanism underlying metformin function in GDM-induced renal dysfunction remains unclear. Since T2DM and GDM 
share similar pathophysiological and genetic characteristics (8), the present study aimed to investigate whether the protective effect of metformin on renal dysfunction in GDM mice is associated with the pro-inflammatory response via the MAPK signaling pathway. In the present study, it was hypothesized that metformin exposure during pregnancy may decrease GDM-induced renal dysfunction.

\section{Materials and methods}

Reagents. Metformin and D-glucose were purchased from Sigma-Aldrich (Merck KGaA, Darmstadt, Germany). The primary antibodies anti-MAPK1/3 (cat. no. 9102; 1:1,000), phosphorylated-MAPK1/3 (p-MAPK1/3; cat. no; 4370, 1:1,000), MAPK8 (cat. no. 9252; 1:1,000), p-MAPK8 (cat. no. 9255; 1:1,000), MAPK14 (cat. no. 9212; 1:1,000), p-MAPK14 (cat. no. 4511; 1:1,000) and GAPDH (cat. no. 8884; 1:1,000) were purchased from Cell Signaling Technology, Inc. (Danvers, MA, USA). The primary antibody anti- $\beta$-actin (cat. no. sc-47778, 1:1,000) was purchased from Santa Cruz Biotechnology, Inc. (Dallas, TX, USA). Horseradish peroxidase-labeled secondary antibodies (cat. no. TA130003, 1:1,000) were purchased from OriGene Technologies, Inc. (Beijing, China). Enhanced chemiluminescence (ECL) reagent was purchased from EMD Millipore (Billerica, MA, USA).

Animals. All procedures involving animal experiments were in accordance with the ethical standards of The Chongqing Medical University Animal Policy and Welfare committee, and ethical approval was obtained from The Ethics Committee of The First Affiliated Hospital of Chongqing Medical University (approval no. 2017-030-2). All efforts were made to minimize animal suffering. A total of 36 female C57BL/6 mice (18-22 g, 7 weeks old) were purchased from The Animal Centre of Chongqing Medical University (Chongqing, China), and acclimatized for 1 week. The mice were housed at $22-24^{\circ} \mathrm{C}$ and $40-60 \%$ humidity, under a 12-h light/dark cycle, with free access to food and water.

In total, 24 8-weeks-old mice were randomly divided into 4 groups ( $n=6$ per group), including the control group, the GDM group and two GDM groups treated with two different doses of metformin (300 and $600 \mathrm{mg} / \mathrm{kg} /$ day). These working concentrations were determined by a series of preliminary experiments (data not shown). The control group was fed a low-fat diet (LFD; Research Diets, Inc., New Brunswick, NJ, USA; cat. no. AIN-93G), which contained $15.8 \%$ fat, $20.3 \%$ proteins and $63.9 \%$ carbohydrates. The mice in the other three groups were fed a high-fat diet (HFD; Research Diets, Inc.; cat. no. D12451), which contained $45 \%$ fat, $20 \%$ proteins and $35 \%$ carbohydrates. Following 1 week of the LFD or HFD, all mice (age, 9 weeks) were mated with male mice (female to male ratio, 2:1) and fed the special diet described above until embryonic day 18.5 (E18.5). GDM mice were treated with the two doses of metformin between E11.5 and E17.5 via gavage, whereas the untreated GDM and control groups were administered PBS via gavage. The oral glucose tolerance test (OGTT) was performed on female mice at E0.5 and E16.5. Urine was collected from each mouse in a metabolic cage at E17.5 to measure the level of albumin in the urine. Mice were sacrificed following $6 \mathrm{~h}$ of fasting at E18.5 by intraperitoneal injection of $1 \%$ pentobarbital sodium $(50 \mathrm{mg} / \mathrm{kg})$ combined with $\mathrm{CO}_{2}$ asphyxiation in a sealed chamber. The chamber was filled at a rate of $20 \% \mathrm{CO}_{2}$ chamber volume $/ \mathrm{min}$. Mice that did not exhibit spontaneous breathing or blink reflex for $>3$ min were considered to be deceased. Serum was collected to measure the circulating levels of certain factors including insulin, $\beta 2-$ microglobulin, IL-6 and TNF- $\alpha$. The kidneys were removed following sacrifice. Half of the left kidney from each mouse was fixed with $4 \%$ paraformaldehyde and then dehydrated in $25 \%$ sucrose solution at $4^{\circ} \mathrm{C}$ for $24 \mathrm{~h}$, respectively, for pathological analysis. The remaining half of each kidney was snap-frozen in liquid nitrogen for western blot analysis.

Evaluation of glucose tolerance and homeostatic model assessment for insulin resistance (HOMA-IR). In order to investigate glucose intolerance, OGTT was performed at E0.5 (the morning that vaginal plug was observed) and E16.5. D-glucose $(2 \mathrm{mg} / \mathrm{g}$ ) was administered to 6-h fasted mice via gavage, and glucose levels were determined following 0, 30, 60,90 and $120 \mathrm{~min}$ at the tail tip with a glucometer (Johnson \& Johnson, New Brunswick, NJ, USA). Glucose tolerance was analyzed by the area under the curve (AUC). HOMA-IR was measured at E18.5. Fasting blood glucose levels were determined in 6-h fasted mice at the tail tip with a glucometer. Serum insulin levels were determined by ELISA. HOMA-IR was calculated using the following formula: HOMA-IR=[fasting blood glucose level (mmol/l) x fasting serum insulin level $(\mathrm{mIU} / \mathrm{l})] / 22.5$ (17).

Determination of albumin levels in the urine and serum biochemical parameters by ELISA. Urine was collected from mice at E17.5 in metabolic cages for $12 \mathrm{~h}$ and stored at $-80^{\circ} \mathrm{C}$. At E18.5, the mice were sacrificed following $6 \mathrm{~h}$ of fasting. Blood was collected by cardiac puncture and immediately centrifuged at $1,000 \mathrm{x} \mathrm{g}$ and $4^{\circ} \mathrm{C}$ for $15 \mathrm{~min}$ to collect serum. Serum samples were stored at $-80^{\circ} \mathrm{C}$. Mouse ELISA kits (Westang BioTech, Inc., Ltd., Shanghai, China) were used to determined the urine albumin (cat. no. N04323) and serum insulin (cat. no. N04572) levels in addition to the circulating levels of $\beta 2$-microglobulin (cat. no. N03915), IL-6 (cat. no. N03981) and TNF- $\alpha$ (cat. no. A05224). Absorbance was measured at $450 \mathrm{~nm}$.

Histology and morphometry. The mice were sacrificed at E18.5 and the kidneys were immediately removed. Half of the left kidney in each mouse was fixed in $4 \%$ paraformaldehyde as aforementioned, embedded in paraffin and 5- $\mu \mathrm{m}$ sections were prepared. Hematoxylin and eosin $(\mathrm{H} \& \mathrm{E})$ and periodic acid-Schiff (PAS) staining were performed using commercial kits (cat. no. G1120 and G1281, Beijing Solarbio Science \& Technology Co., Ltd, Beijing, China). Briefly, H\&E staining was using hematoxylin for $5 \mathrm{~min}$ followed by eosin for $2 \mathrm{~min}$ at room temperature; PAS was stained with Schiff Reagent for $15 \mathrm{~min}$ and hematoxylin for $1 \mathrm{~min}$ at room temperature. H\&E and PAS stained slides were observed using a light microscope (OLYMPUS X81; Olympus Corporation, magnification, x400).

Western blot analysis. Protein expression levels of MAPK1/3, -8 and -14 , and p-MAPK1/3, -8 and -14 were determined by western blotting. Kidney tissue samples ( $n=3$ per group) were 
Table I. Characteristics of the control and GDM groups.

\begin{tabular}{lrr}
\hline Measurements & Control & GDM model \\
\hline Blood glucose at $0 \mathrm{~min}, \mathrm{mmol} / \mathrm{l}$ & $4.92 \pm 0.46$ & $5.62 \pm 0.38^{\mathrm{a}}$ \\
Blood glucose at $30 \mathrm{~min}, \mathrm{mmol} / \mathrm{l}$ & $13.87 \pm 0.82$ & $17.00 \pm 0.68^{\mathrm{c}}$ \\
Blood glucose at $60 \mathrm{~min}, \mathrm{mmol} / \mathrm{l}$ & $11.15 \pm 0.83$ & $12.87 \pm 0.43^{\mathrm{b}}$ \\
Blood glucose at $90 \mathrm{~min}, \mathrm{mmol} / \mathrm{l}$ & $7.18 \pm 0.44$ & $9.55 \pm 0.39^{\mathrm{c}}$ \\
Blood glucose at $120 \mathrm{~min}, \mathrm{mmol} / \mathrm{l}$ & $5.15 \pm 0.24$ & $5.51 \pm 0.27^{\mathrm{a}}$ \\
Area under the curve & $1,117.17 \pm 48.59$ & $1,356.33 \pm 22.33^{\mathrm{c}}$ \\
E0.5 body weight, g & $22.17 \pm 0.30$ & $22.29 \pm 0.54$ \\
E18.5 body weight, g & $33.28 \pm 0.66$ & $38.45 \pm 0.57^{\mathrm{c}}$ \\
E18.5 fasting glucose, mmol/1 & $5.12 \pm 0.19$ & $5.68 \pm 0.42^{\mathrm{b}}$ \\
E18.5 fasting insulin, mmol/1 & $18.32 \pm 0.90$ & $21.97 \pm 0.31^{\mathrm{c}}$ \\
E18.5 HOMA-IR & $4.17 \pm 0.16$ & $5.55 \pm 0.21^{\mathrm{c}}$ \\
\hline
\end{tabular}

Data are presented as the mean \pm deviation. ${ }^{\mathrm{a}} \mathrm{P}<0.05,{ }^{\mathrm{b}} \mathrm{P}<0.01$ and ${ }^{\mathrm{c}} \mathrm{P}<0.001$ vs. control group. GDM, gestational diabetes mellitus; E, embryonic day.

homogenized in radioimmunoprecipitation assay solution containing 1:100 phosphatase inhibitor cocktail (phenylmethylsulfonyl fluoride, cat. no. ST506, Beyotime Beyotime Institute of Biotechnology, Inc., Shanghai, China). Protein concentration was determined using the bicinchoninic acid assay kit (Beyotime Institute of Biotechnology, Inc.). Protein lysates $(50 \mu \mathrm{g})$ were loaded in each lane, separated by $10 \%$ SDS-PAGE and transferred onto polyvinylidene fluoride membranes (Bio-Rad Laboratories, Inc., Hercules, CA, USA) at $100 \mathrm{~V}$ for $2 \mathrm{~h}$. Membranes were blocked with 5\% non-fat milk in TBST $(0.075 \%$ Tween) for $1 \mathrm{~h}$ at room temperature, and sequentially incubated with primary antibodies (dilution, 1:1,000) at $4^{\circ} \mathrm{C}$ overnight and horseradish peroxidase-conjugated secondary antibodies (dilution, 1:5,000) at room temperature for $1 \mathrm{~h}$. Bands were visualized with an ECL reagent and quantitated using ImageJ v1.8.0 (National Institutes of Health, Bethesda, MD, USA). Data were normalized to $\beta$-actin or GAPDH levels.

Statistical analysis. Data are presented as the mean \pm standard error. Comparisons among four or three groups were performed by one-way analysis of variance, followed by the least significant difference multiple-comparison post hoc test. Bivariate correlation analysis was used to analyze the correlation between the levels of inflammatory cytokines and symptoms of renal dysfunction. $\mathrm{P}<0.05$ was considered to indicate a statistically significant difference. Data were analyzed using the SPSS statistical package (version 17.0; SPSS, Inc., Chicago, IL, USA).

\section{Results}

Establishment of a HFD-induced GDM model. At E0.5, body weight was measured, and blood glucose levels and glucose tolerance were determined via OGTT, and no significant differences between the two groups were observed (data not shown). The OGTT was subsequently performed at E16.5. Blood glucose levels in the GDM group at 0,30, 60, 90 and $120 \mathrm{~min}$ and the AUC value representing glucose tolerance were significantly increased compared with the control group (Table I). The present data suggested that glucose tolerance in the GDM group was impaired. Additionally, pregnant mice at E18.5 were weighed and HOMA-IR was calculated. Body weight in the GDM group was significantly increased compared with the control group (Table I). The levels of glucose, insulin and HOMA-IR were significantly increased in the GDM group compared with the control group (Table I), suggesting that insulin sensitivity was impaired in GDM mice. The present results suggested that the established mouse model exhibited reliable symptoms of GDM. In addition, to investigate the effects of GDM in middle- to old-aged animals, OGTT and the AUC of OGTT were performed at 3 weeks following delivery, and no significant differences between the two groups were observed (Table II), which suggested a GDM model was induced rather than T2DM.

Metformin attenuates glucose tolerance and insulin resistance in GDM mice during pregnancy. Body weight (Fig. 1A), blood glucose levels at all time points (Fig. 1B) and glucose tolerance (Fig. 1C) in the GDM group at E18.5 were increased compared with the control group. Treatment with metformin at two concentrations (300 and $600 \mathrm{mg} / \mathrm{kg} /$ day) significantly reversed these effects. The levels of blood glucose, serum insulin and HOMA-IR (Fig. 1D-F) were significantly increased in the fasting mice of the GDM group compared with the control group (Table I). Treatment with metformin at the two concentrations tested significantly decreased the levels of blood glucose, insulin secretion and HOMA-IR compared to untreated GDM mice, in a dose-dependent manner (Fig. 1D-F).

Metformin attenuates GDM-induced renal dysfunction during pregnancy. The mice were sacrificed at E18.5 and the kidneys were harvested. The kidney-to-body weight ratios were measured and no significant differences among the four groups were observed (data not shown). Subsequently, the markers of early renal dysfunction were investigated by ELISA. The levels of urine albumin (Fig. 2A) and 
Table II. OGTT results and the AUC of OGTT at 3 weeks following delivery in the control and GDM groups.

\begin{tabular}{lrr}
\hline Measurements & Control & GDM model \\
\hline Blood glucose at $0 \mathrm{~min}, \mathrm{mmol} / \mathrm{l}$ & $5.73 \pm 0.22$ & $5.20 \pm 0.30$ \\
Blood glucose at $30 \mathrm{~min}, \mathrm{mmol} / \mathrm{l}$ & $10.33 \pm 0.79$ & $11.84 \pm 0.77$ \\
Blood glucose at $60 \mathrm{~min}, \mathrm{mmol} / \mathrm{l}$ & $7.15 \pm 0.73$ & $7.20 \pm 0.35$ \\
Blood glucose at $90 \mathrm{~min}, \mathrm{mmol} / \mathrm{l}$ & $6.13 \pm 0.14$ & $6.16 \pm 0.15$ \\
Blood glucose at $120 \mathrm{~min}, \mathrm{mmol} / \mathrm{l}$ & $5.78 \pm 0.17$ & $5.42 \pm 0.22$ \\
AUC & $880.5 \pm 30.77$ & $915.4 \pm 36.38$
\end{tabular}

Data are presented as the mean \pm deviation. AUC, area under the curve; GDM, gestational diabetes mellitus; OGTT, oral glucose tolerance test.
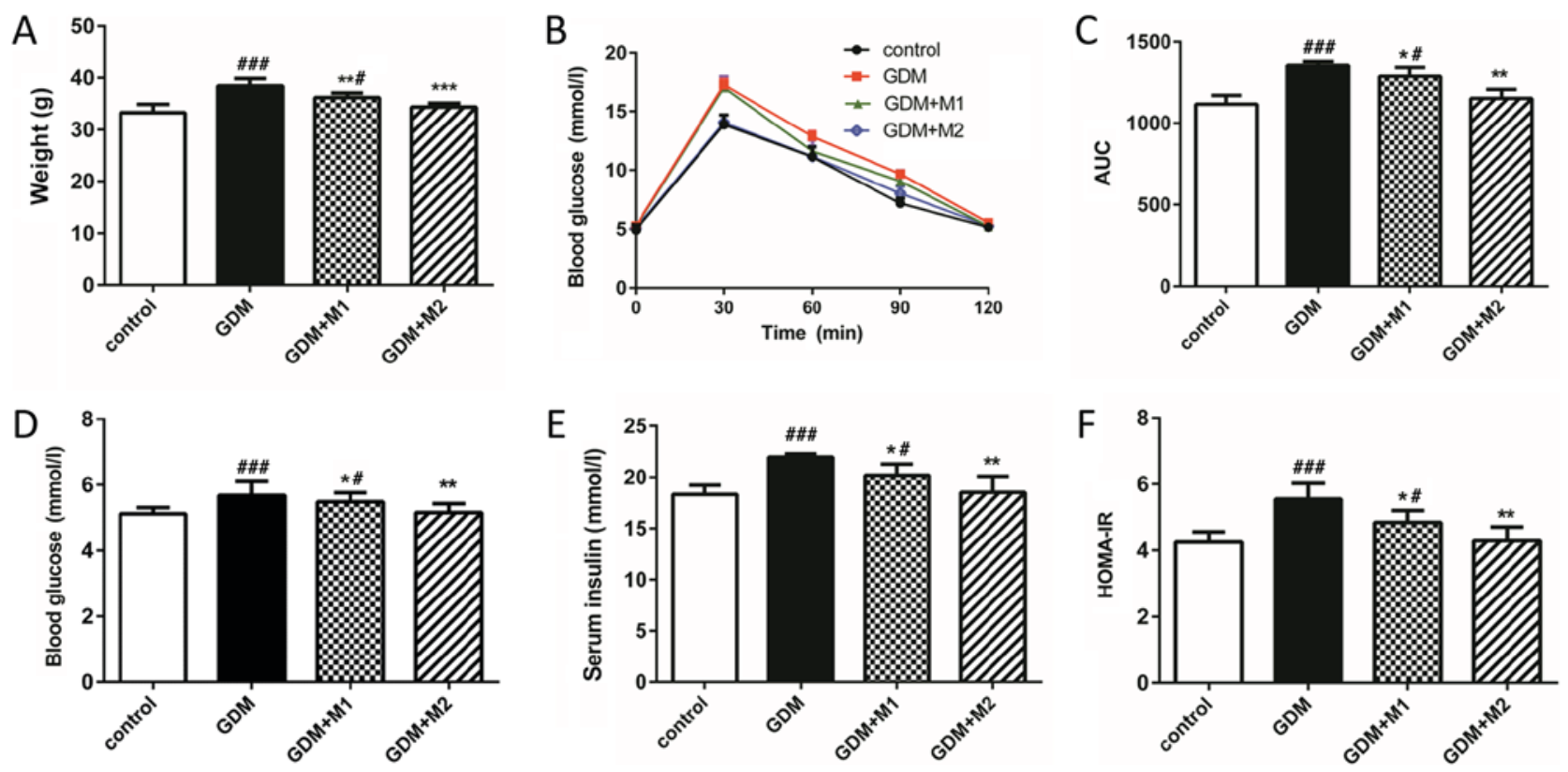

Figure 1. Metformin attenuates glucose tolerance and insulin resistance in GDM mice during pregnancy. (A) Body weight in the four groups at E18.5. (B) Blood glucose levels in the four groups at E16.5, as assessed by OGTT. (C) AUC analysis of the OGTT represents glucose tolerance. (D) Fasting blood glucose levels in the four groups at E18.5. (E) Fasting serum insulin levels in the four groups determined by ELISA at E18.5. (F) HOMA-IR in the four groups at E18.5. ${ }^{*} \mathrm{P}<0.05,{ }^{* *} \mathrm{P}<0.01$ and ${ }^{* * * *} \mathrm{P}<0.001$ vs. GDM group; ${ }^{*} \mathrm{P}<0.05$ and ${ }^{\# \# \#} \mathrm{P}<0.001$ vs. control group. M1, metformin at $300 \mathrm{mg} / \mathrm{kg} / \mathrm{day} ; \mathrm{M} 2$, metformin at $600 \mathrm{mg} / \mathrm{kg} /$ day; E, embryonic day; GDM, gestational diabetes mellitus; HOMA-IR, homeostatic model assessment for insulin resistance; AUC, area under the curve; OGTT, oral glucose tolerance test.

serum $\beta 2$-microglobulin (Fig. 2B) in the GDM group were significantly increased compared with control mice. However, treatment with metformin (300 and $600 \mathrm{mg} / \mathrm{kg} /$ day) significantly reversed these effects. Additionally, H\&E (Fig. 2C) and PAS (Fig. 2D) staining were performed to assess kidney histomorphology. The glomerular diameters following H\&E staining and the basement membrane thicknesses following PAS staining of the four groups of mice were measured. The glomerular volume in the GDM group was increased compared with the control group. Basement membrane thickening and mesangial matrix proliferation was observed in the GDM group. The present alterations observed by H\&E and PAS staining were markedly reversed by treatments with metformin (300 and $600 \mathrm{mg} / \mathrm{kg} /$ day). These results suggested that the markers of early renal dysfunction were significantly increased in the GDM group and dose-dependently reduced by metformin treatment, which confirmed the protective effect of metformin.
Inflammation and the MAPK pathway are associated with the protective effect of metformin on renal dysfunction in GDM during pregnancy. The concentrations of IL- 6 and TNF- $\alpha$ in the serum of pregnant mice at E18.5 were determined by ELISA. Serum levels of IL-6 (Fig. 3A) and TNF- $\alpha$ (Fig. 3B) in the GDM group were significantly increased compared with the control group, and metformin at $600 \mathrm{mg} / \mathrm{kg} /$ day reversed these effects. The two metformin doses significantly decreased the serum levels of IL-6 (Fig. 3A). However, treatment with metformin at $300 \mathrm{mg} / \mathrm{kg} /$ day was not sufficient to significantly decrease the levels of TNF- $\alpha$; this effect may be due to the small sample size (Fig. 3B).

The activity of the MAPK signaling pathway in renal tissue at E18.5 was assessed by western blotting. No differences were observed in the total protein levels of MAPK14, -1/3 and -8 among the four groups (data not shown). The phosphorylation levels of MAPK1/3 (Fig. 3C), 14 (Fig. 3D), and 8 (Fig. 3E) were increased in the GDM group, and both doses of metformin 
A
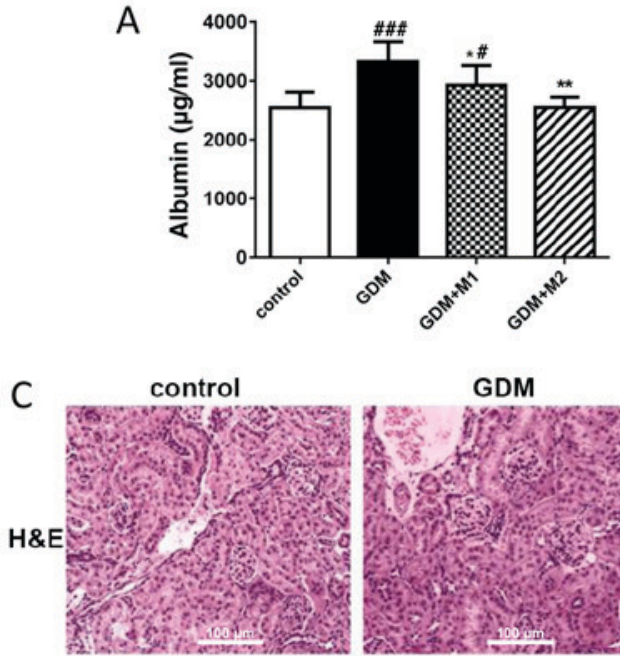

D

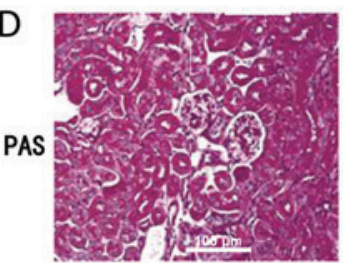

GDM
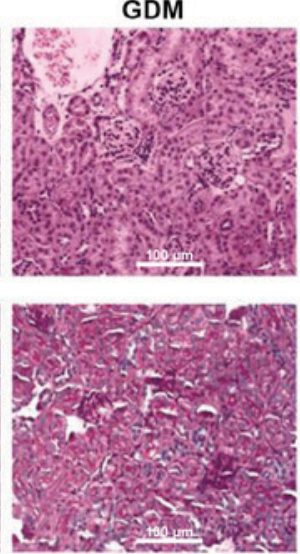

$\mathrm{GDM}+\mathrm{M} 1$
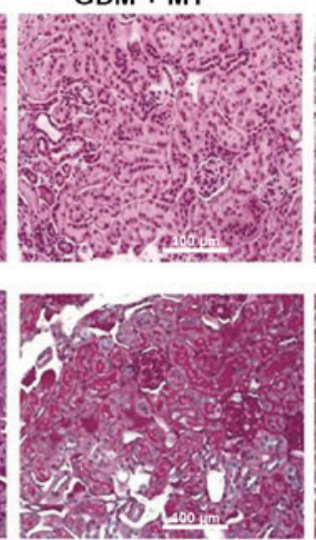

B

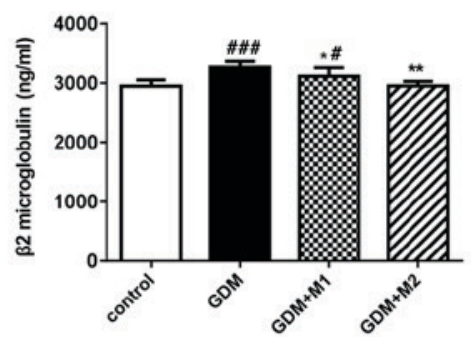

$\mathrm{GDM}+\mathrm{M} 2$
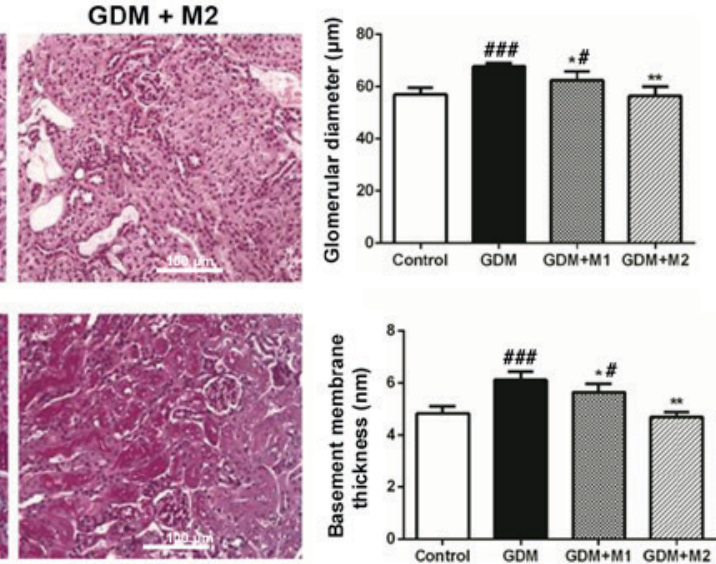

Figure 2. Metformin attenuates renal dysfunction in the GDM groups during pregnancy. (A) Level of urine albumin at E17.5 determined by ELISA. (B) Serum 32-microglobulin levels at E18.5 determined by ELISA. (C) H\&E and (D) PAS staining of the kidney tissue at E18.5. Magnification, x400; scale bar, $100 \mu$ m. The GDM group exhibited increased glomerulus volume, as assessed by H\&E staining, and glomerular basement membrane thickening and mesangial matrix expansion, as determined by PAS staining. Treatment with metformin reversed these effects. $\mathrm{n}=6$ per group; ${ }^{*} \mathrm{P}<0.05$ and ${ }^{* *} \mathrm{P}<0.01$ vs. GDM group; ${ }^{\#} \mathrm{P}<0.05$

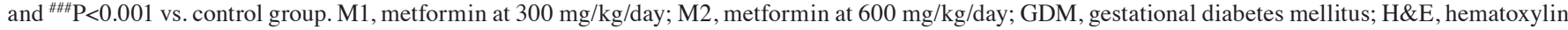
and eosin; PAS, periodic acid-Schiff.

(300 and $600 \mathrm{mg} / \mathrm{kg} /$ day) significantly reversed these effects. Metformin at a concentration of $600 \mathrm{mg} / \mathrm{kg} /$ day was sufficient to decrease the phosphorylation levels of MAPKs in GDM mice to a similar level as the control.

High levels of serum $\beta 2$-microglobulin (18) and urine albumin (19) are symptoms of renal dysfunction. Therefore, the association between the serum levels of cytokines and the symptoms of renal dysfunction was assessed. The present results suggested that the serum levels of IL-6 and TNF-a were positively correlated with the levels of $\beta 2$-microglobulin and albumin (Fig. 4). The present results indicated that the protective effect of metformin on renal dysfunction in the GDM group may be associated with the MAPK signaling pathway and the pro-inflammatory response.

\section{Discussion}

GDM, defined as diabetes diagnosed during pregnancy, is associated with various adverse outcomes affecting the mother and the newborn, including perinatal mortality, congenital malformations, macrosomia, shoulder dystocia, birth injuries and neonatal complications (2). The majority of women who develop GDM are normoglycemic following delivery (20); however, they are more susceptible to developing cardiovascular diseases (21) and T2DM (5) postpartum. Although a number of studies demonstrated the complications associated with GDM (2-4), the effects of GDM on the function of the kidneys during pregnancy and following delivery remain unclear. The present findings suggested that treatment with metformin during pregnancy exhibited a protective effect on renal dysfunction during pregnancy. Inflammation may be associated with renal dysfunction in GDM mice, and the MAPK signaling pathway may be involved in the protective effect of metformin on renal dysfunction in GDM mice.

A novel model of GDM was developed in the present study. C57BL/6J female mice were fed a HFD and the control group was fed a LFD between 1 week prior to mating and E18.5. The GDM mouse model exhibited increased body weight, glucose intolerance and insulin resistance; all symptoms observed in human GDM. The majority of the GDM models are induced by treatment with streptozotocin (STZ) (22) or by a diet high in fat and glucose. The GDM model induced by STZ exhibits irreversible necrosis of pancreatic $\beta$ cells, a phenotype observed in type 1 diabetes, and so it may not be a reliable model to study T2DB (23). The GDM model induced by a diet high in fat and glucose may result in diabetes mellitus prior to pregnancy (24). The increased glucose levels in these models may not decrease following delivery (25), suggesting that these models may not be suitable for investigating the impact of GDM on kidney function. By contrast, the novel GDM model generated in the present study is characterized by a controlled diet at early pregnancy (E0.5). At E0.5, the body weight, blood glucose and glucose tolerance, assessed by OGTT, exhibited no significant differences between the GDM and control groups. The present model may represent a suitable and reliable model for the study of GDM in middleto old-aged animals and in the investigation of the impact of GDM on the offspring. To the best of the authors' knowledge, 

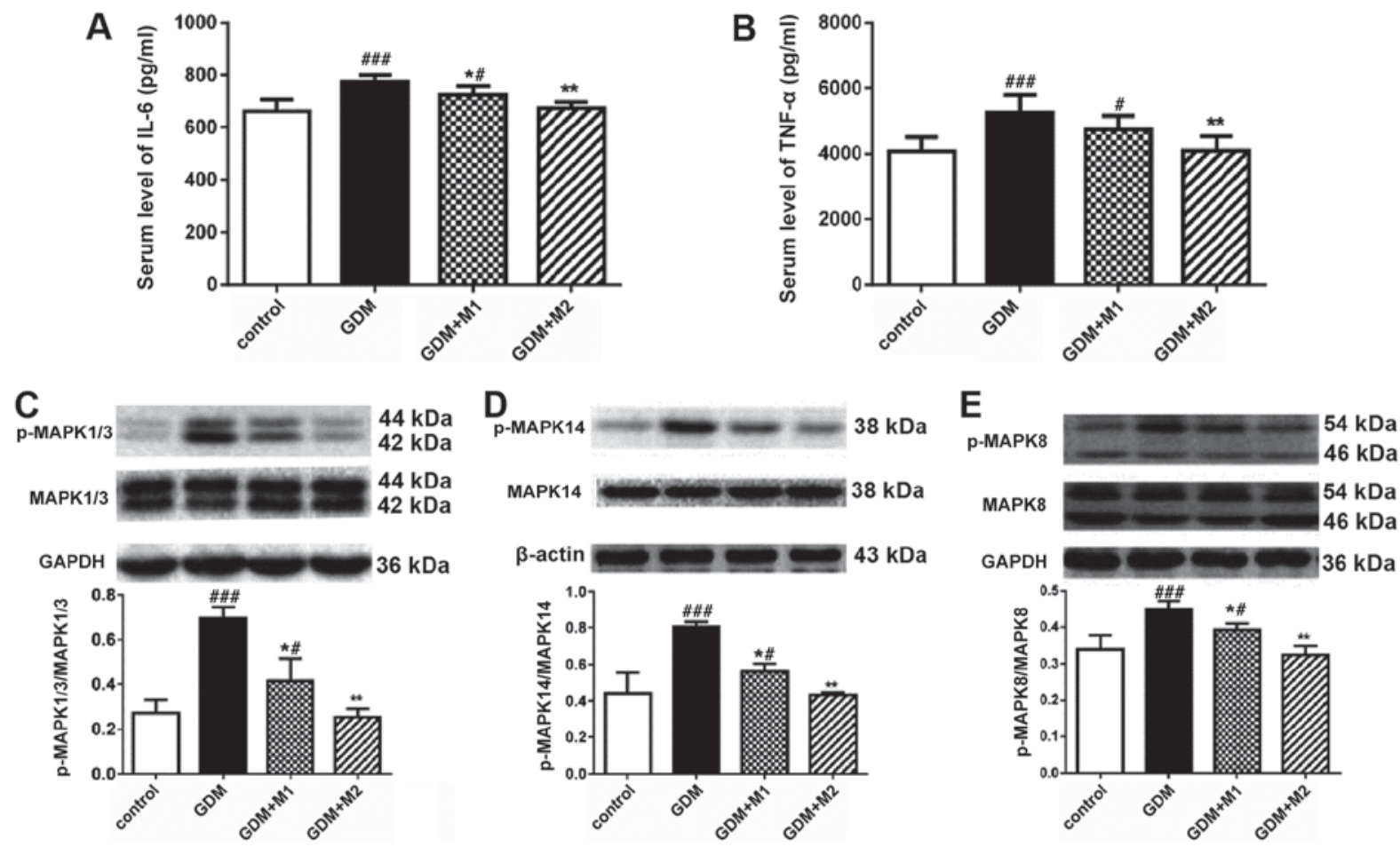

Figure 3. Inflammatory response and the MAPK pathway are involved in the protective effect of metformin on renal dysfunction in GDM during pregnancy. Serum levels of (A) IL-6 and (B) TNF- $\alpha$ at E18.5 were analyzed by ELISA. Protein expression levels of (C) p-MAPK1/3,(D) p-MAPK14 and (E) p-MAPK8 are presented as the ratio between the p-protein and the respective total protein. Protein expression levels of MAPKs in the kidney tissue at E18.5 were determined by western blotting. $\mathrm{n}=6$ per group; ${ }^{*} \mathrm{P}<0.05$ and ${ }^{* *} \mathrm{P}<0.01$ vs. GDM group; ${ }^{\#} \mathrm{P}<0.05$ and ${ }^{\# \# \#} \mathrm{P}<0.001$ vs. control group. GDM, gestational diabetes mellitus; TNF- $\alpha$, tumor necrosis factor- $\alpha$; IL-6, interleukin 6; MAPK, mitogen-activated protein kinase; p-, phosphorylated.

the present study is the first one using this model of GDM. Antidiabetic drugs are frequently used when altering the diet and increasing exercise fails to decrease the levels of blood glucose in women with GDM. The short-term safety of these compounds in the mother and in the fetus were confirmed in various previous studies (14-16). Metformin is able to cross the placental barrier and may potentially pose a threat to the fetus; however, metformin in a gestational diabetes trial (26) demonstrated that there were no adverse outcomes in a 2-year follow-up study of infants whose mother was administered metformin to treat GDM. The American College of Obstetrics and Gynecology (27) in 2013 and the International Federation of Gynecology and Obstetrics (28) in 2015 recommended metformin as the first-line anti-diabetic agent in women with GDM. Furthermore, metformin may have a potential protective effect on insulin resistance in the newborn (26). An additional advantage of metformin in the treatment of GDM is the low risk of inducing hypoglycemia and weight loss, whereas insulin, an alternative treatment for GDM, promotes weight gain, which may be a risk factor for the development of T2DM (20). In the present study, the two doses of metformin tested (300 and $600 \mathrm{mg} / \mathrm{kg} /$ day) reversed the increased weight gain, and impaired glucose tolerance and insulin resistance in GDM mice, in a dose-dependent manner.

A number of previous studies demonstrated that GDM may exhibit adverse effects on renal function. Friedman et al (13) observed a higher risk of microalbuminuria in women with a history of GDM. Bomback et al (29) demonstrated that GDM, without subsequent T2DM, is a risk factor for the development of microalbuminuria. These previous studies suggested that GDM may pose a risk for kidney function. In the present study, renal dysfunction was examined in GDM mice at E18.5 by determining the levels of urine albumin and serum $\beta 2$-microglobulin, and $\mathrm{H} \& \mathrm{E}$ and PAS staining were performed to investigate the morphology of kidney tissues. Urine albumin, a marker of impaired glomerular filtration rate, is considered a marker of early DN (30). The American Diabetes Association has recommended annual testing for the levels of urine albumin in patients with T2DM to identify chronic renal disease (31). Additionally, serum $\beta 2$-microglobulin is considered a marker of renal dysfunction (32). The present study identified that the levels of urine albumin and serum $\beta 2$-microglobulin were significantly increased in the GDM group during pregnancy, indicating that high levels of urine albumin and serum $\beta 2$-microglobulin may be reliable markers for early renal dysfunction. An increase in glomerular size, glomerular basement membrane thickening and mesangial matrix expansion was detected in the GDM group, as assessed by H\&E and PAS staining. The present results suggested that treatment with metformin during pregnancy exhibited a protective effect on renal function during pregnancy.

DN, a severe complication of T2DM, is associated with MAPKs (10) and pro-inflammatory signaling pathways (11); however, a limited number of previous studies investigated the mechanism underlying GDM-induced renal dysfunction. A previous study demonstrated that GDM is characterized by a state of chronic, low-grade inflammation, which is induced by various metabolites and nutrients, and is associated with increased insulin resistance (33). IL-6 is 
A Correlation between IL-6 and $\beta 2$-microglobulin

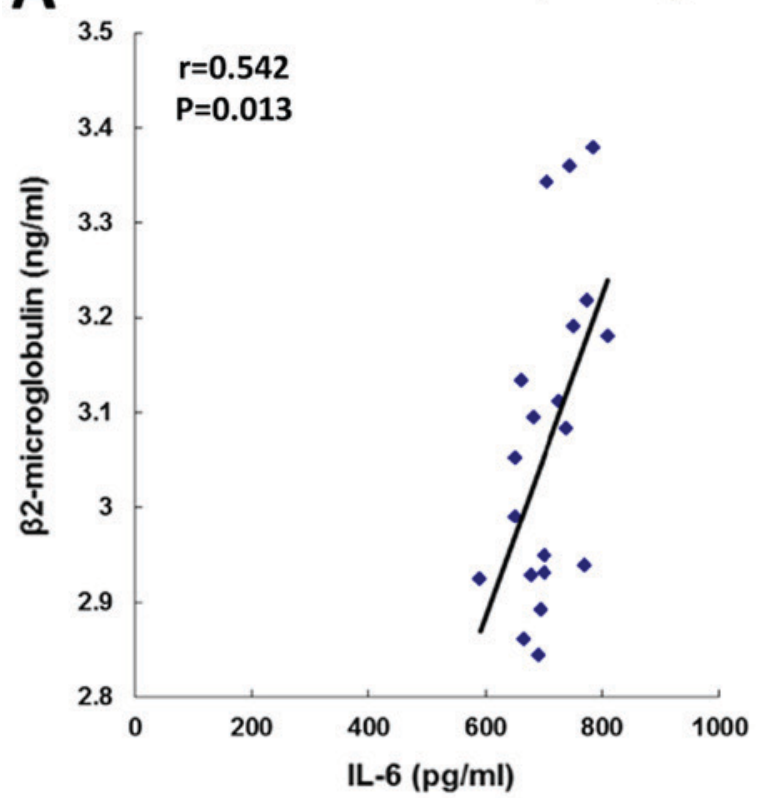

\section{Correlation between IL-6 and albumin}

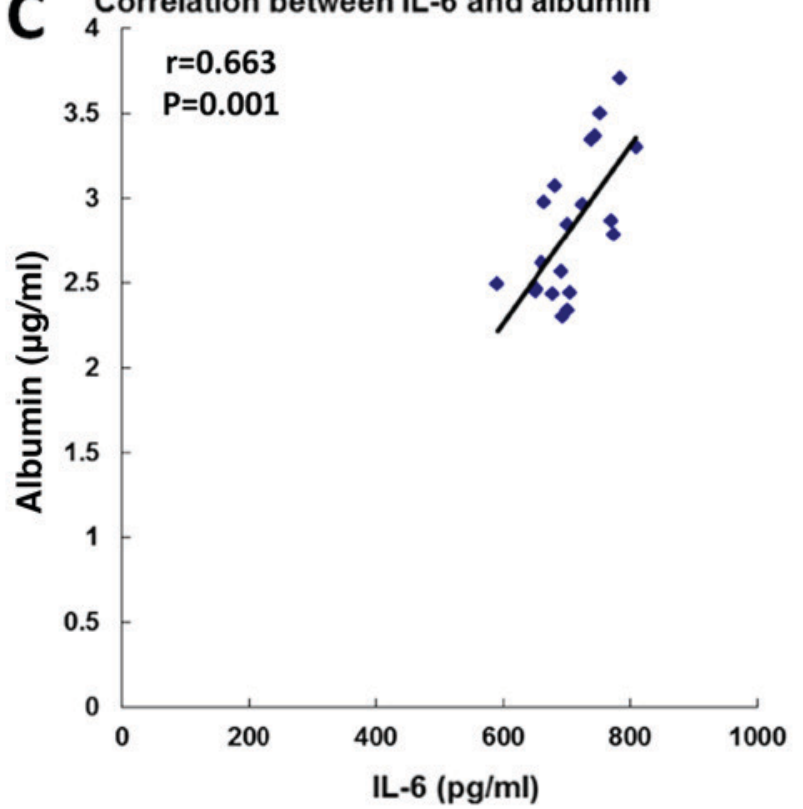

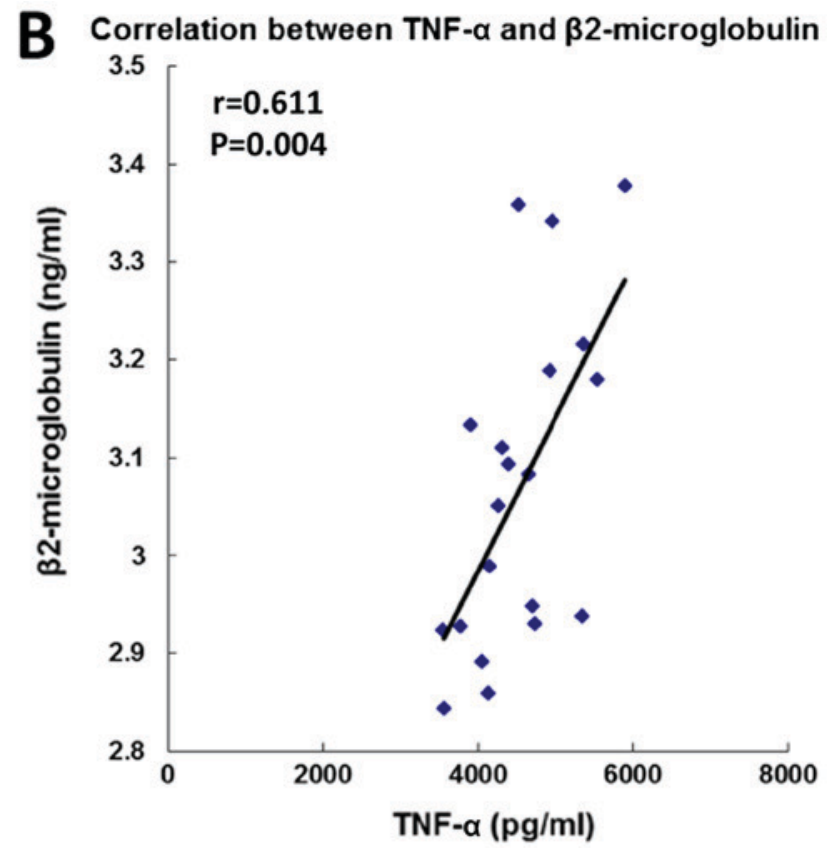

D Correlation between TNF- $\alpha$ and albumin

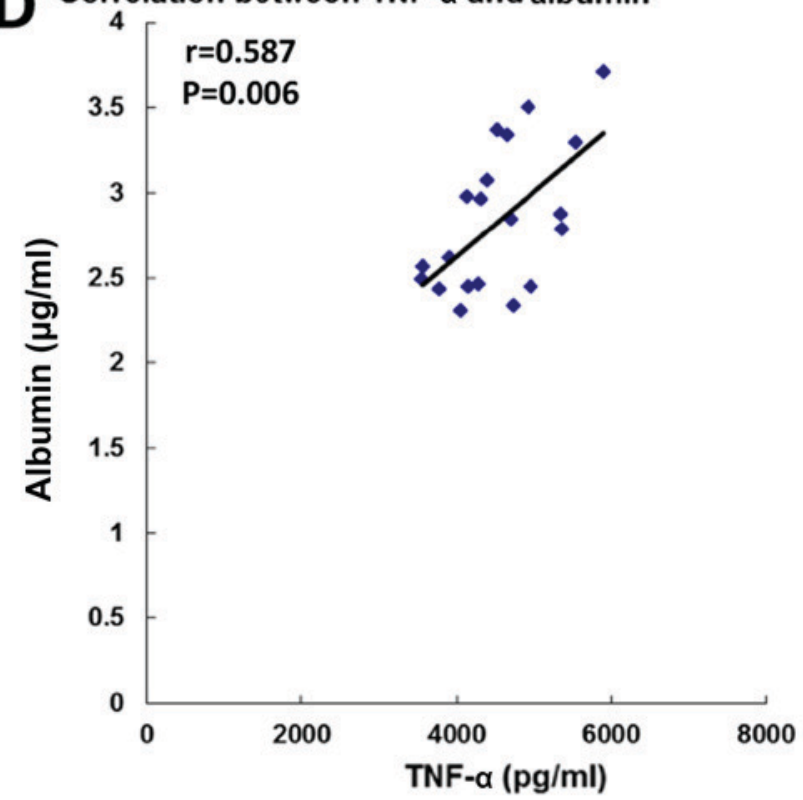

Figure 4. Correlation between the serum levels of cytokines and symptoms of renal dysfunction. (A) IL-6 and (B) TNF- $\alpha$ levels were positively correlated with serum levels of $\beta 2$-microglobulin. (C) IL-6 and (D) TNF- $\alpha$ levels were positively correlated with urine levels of albumin. TNF- $\alpha$, tumor necrosis factor- $\alpha$; IL-6, interleukin 6.

an important pro-inflammatory cytokine associated with impaired glucose tolerance (34). Additionally, TNF- $\alpha$ was identified in a previous study to be associated with inflammation and insulin resistance in GDM (35). In the present study, the association between the protective effect of metformin on renal dysfunction in GDM mice and these inflammatory cytokines was investigated. The MAPK signaling pathway consists of MAPK1/3, -14 and -8, and it is essential for the regulation of inflammation (36). Since GDM may be a predictor of T2DM (5), and MAPKs serve important roles in the development of DN by regulating inflammatory cytokines (10), the involvement of the MAPK pathway in the protective effects of metformin on renal dysfunction in GDM mice was examined in the present study. Serum levels of IL-6 and TNF- $\alpha$ in the GDM group were increased at E18.5, suggesting that these cytokines may be associated with renal dysfunction. Furthermore, in the present study, the phosphorylation levels of MAPK1/3, -14 and -8 were identified to be increased in the GDM group at E18.5, and metformin was able to decrease the protein expression level of phosphorylated MAPKs in a dose-dependent manner.

A previous study demonstrated that high glucose treatment of monocytic cells caused the upregulation of 41 genes and the downregulation of 15 genes, including chemokines, cytokines, chemokines receptors, adhesion molecules and 
integrins (37). In addition, compared with the normal glucose group $(<5.5 \mathrm{mmol} / \mathrm{l})$, HUVECs treated with $50 \mathrm{mmol} / 1$ glucose exhibited an increase in the protein expression levels of TNF- $\alpha$ and IL-6 (38). In line with these in vitro data, an impaired balance between circulating pro- and anti-inflammatory cytokines was observed in patients with gestational diabetes (39). Collectively, the present results and those of previous studies suggested that the MAPK pathway may be involved in the protective effect of metformin on renal dysfunction in GDM mice. Metformin is able to decrease microglia-mediated inflammation via the nuclear factor (NF)- $\kappa$ B and MAPK signaling pathways to improve neurobehavioral function following traumatic brain injury (40). In cancer, metformin was identified to affect various signaling pathways, including adenosine monophosphate-activated protein kinase, mammalian target of rapamycin, insulin-like growth factor, MAPK, human epidermal growth factor receptor-2, and $\mathrm{NF}-\kappa \mathrm{B}$ pathways (41). Previous studies demonstrated that metformin mediated the activation of an insulin-dependent pathway via the upregulation of protein kinase $\mathrm{B}$ serine/threonine kinase 1 in the treatment of T2DM in human endometrial cells $(42,43)$.

The present study presented various limitations. Although the symptoms of GDM in the generated model are similar to those in human patients with GDM, and certain parameters associated with early renal dysfunction were detected in the experimental group, renal dysfunction may occur only in a subset of GDM mice. Polymerase chain reaction and in situ immunostaining analyses were not performed in the present study and may represent useful methods to validate the GDM model. Furthermore, the systemic effects of GDM may involve multiple pathways and molecular mechanisms, and the present study focused on the effects of GDM on kidney function and the MAPK signaling pathway. Further studies are required to validate the GDM model, and additional studies may identify multiple mechanisms and signaling pathways associated with the novel mouse model. Furthermore, although GDM was induced in C57BL/6 mice, the translational potential of these findings remains to be confirmed.

In conclusion, the present study suggested that GDM-induced renal dysfunction during late pregnancy was reversed by treatment with metformin. The effect of metformin was dose-dependent, and $600 \mathrm{mg} / \mathrm{kg} /$ day administered between E11.5 and E17.5 may be the optimal dose to treat GDM mice. In addition, the inflammatory cytokines IL- 6 and TNF- $\alpha$ were identified to be associated with renal dysfunction in GDM mice, and the MAPK signaling pathway may be involved in the protective effect of metformin on renal dysfunction in GDM mice.

\section{Acknowledgements}

Not applicable.

\section{Funding}

The present study was funded by The Overseas Expertise Introduction Project for Discipline Innovation [111 Project; grant no. (2017) 175].

\section{Availability of data and materials}

The datasets used and/or analyzed during the current study are available from the corresponding author on reasonable request.

\section{Authors' contributions}

ZL performed the experiments and wrote the manuscript. CT and XY supervised the present study. XY developed the novel mouse model of gestational diabetes mellitus. CT, XY and HQ developed the protocols and designed the study. All authors reviewed the results and approved the final version of the manuscript.

\section{Ethics approval and consent to participate}

All procedures performed in studies involving animals were in accordance with the ethical standards of The Chongqing Medical University Animal Policy and Welfare Committee. Ethical approval was obtained from The Ethics Committee of The First Affiliated Hospital of Chongqing Medical University prior to the commencement of the present study (approval no. 2017-030-2).

\section{Patient consent for publication}

Not applicable.

\section{Competing interests}

The authors declare that they have no competing interests.

\section{References}

1. Weinert LS: International association of diabetes and pregnancy study groups recommendations on the diagnosis and classification of hyperglycemia in pregnancy: Comment to the International Association of Diabetes and Pregnancy Study Groups Consensus Panel. Diabetes Care 33: 676-682, 2010.

2. Mitanchez D: Foetal and neonatal complications in gestational diabetes: Perinatal mortality, congenital malformations, macrosomia, shoulder dystocia, birth injuries, neonatal complications. Diabetes Metab 36: 617-627, 2010.

3. Mitanchez D, Burguet A and Simeoni U: Infants born to mothers with gestational diabetes mellitus: Mild neonatal effects, a long-term threat to global health. J Pediatr 164: 445-450, 2014.

4. Wright CS, Rifas-Shiman SL, Rich-Edwards JW, Taveras EM, Gillman MW and Oken E: Intrauterine exposure to gestational diabetes, child adiposity, and blood pressure. Am J Hypertens 22: 215-220, 2009.

5. Bellamy L, Casas JP, Hingorani AD and Williams D: Type 2 diabetes mellitus after gestational diabetes: A systematic review and meta-analysis. Lancet 373: 1773-1779, 2009.

6. Retnakaran R, Qi Y, Sermer M, Connelly PW, Hanley AJ and Zinman B: Beta-cell function declines within the first year postpartum in women with recent glucose intolerance in pregnancy. Diabetes Care 33: 1798-1804, 2010.

7. Kramer CK, Swaminathan B, Hanley AJ, Connelly PW, Sermer M, Zinman B and Retnakaran R: Each degree of glucose intolerance in pregnancy predicts distinct trajectories of beta-cell function, insulin sensitivity, and glycemia in the first 3 years postpartum. Diabetes Care 37: 3262-3269, 2014.

8. Buchanan TA and Xiang AH: Gestational diabetes mellitus. J Clin Invest 115: 485-491, 2005.

9. Bergrem $\mathrm{H}$ and Leivestad T: Diabetic nephropathy and end-stage renal failure: The Norwegian story. Adv Ren Replace Ther 8: 4-12, 2001. 
10. Wong CK, Ho AW, Tong PC, Yeung CY, Kong AP, Lun SW, Chan JC and Lam CW: Aberrant activation profile of cytokines and mitogen-activated protein kinases in type 2 diabetic patients with nephropathy. Clin Exp Immunol 149: 123-131, 2007.

11. Tuttle KR: Linking metabolism and immunology: Diabetic nephropathy is an inflammatory disease. J Am Soc Nephrol 16 1537-1538, 2005

12. Beharier O, Shoham-Vardi I,Pariente G, Sergienko R, Kessous R, Baumfeld Y, Szaingurten-Solodkin I and Sheiner E: Gestationa diabetes mellitus is a significant risk factor for long-term maternal renal disease. J Clin Endocrinol Metab 100: 1412-1416, 2015.

13. Friedman S, Rabinerson D, Bar J, Erman A, Hod M, Kaplan B, Boner G and Ovadia J: Microalbuminuria following gestational diabetes. Acta Obstet Gynecol Scand 74: 356-360, 1995.

14. Ainuddin J, Karim N, Hasan AA and Naqvi SA: Metformin versus insulin treatment in gestational diabetes in pregnancy in a developing country: A randomized control trial. Diabetes Res Clin Pract 107: 290-299, 2015.

15. Barrett HL, Gatford KL, Houda CM, De Blasio MJ, McIntyre HD, Callaway LK, Dekker Nitert M, Coat S, Owens JA, Hague WM and Rowan JA: Maternal and neonatal circulating markers of metabolic and cardiovascular risk in the metformin in gestational diabetes $(\mathrm{MiG})$ trial: Responses to maternal metformin versus insulin treatment. Diabetes Care 36: 529-536, 2013.

16. Rowan JA, Hague WM, Gao W, Battin MR and Moore MP; MiG Trial Investigators: Metformin versus insulin for the treatment of gestational diabetes. N Engl J Med 358: 2003-2015, 2008.

17. Matthews DR, Hosker JP, Rudenski AS, Naylor BA, Treacher DF and Turner RC: Homeostasis model assessment: Insulin resistance and beta-cell function from fasting plasma glucose and insulin concentrations in man. Diabetologia 28: 412-419, 1985.

18. Lapsley M, Flynn FV and Sansom PA: Beta 2-glycoprotein-1 (apolipoprotein $\mathrm{H}$ ) excretion and renal tubular malfunction in diabetic patients without clinical proteinuria. J Clin Pathol 46 : $465,1993$.

19. Berrut G, Bouhanick B, Fabbri P, Guilloteau G, Bled F, Le Jeune JJ, Fressinaud P and Marre M: Microalbuminuria as a predictor of a drop in glomerular filtration rate in subjects with non-insulin-dependent diabetes mellitus and hypertension. Clin Nephrol 48: 92-97, 1997.

20. Lobner K, Knopff A, Baumgarten A, Mollenhauer U, Marienfeld S, Garrido-Franco M, Bonifacio E and Ziegler AG: Predictors of postpartum diabetes in women with gestational diabetes mellitus. Diabetes 55: 792-797, 2006.

21. Kessous R, Shoham-Vardi I, Pariente G, Sherf M and Sheiner E: An association between gestational diabetes mellitus and long-term maternal cardiovascular morbidity. Heart 99: $1118-1121,2013$

22. Jawerbaum A and White V: Animal models in diabetes and pregnancy. Endocr Rev 31: 680-701, 2010.

23. Caluwaerts S, Holemans K, van Bree R, Verhaeghe J and Van Assche FA: Is low-dose streptozotocin in rats an adequate mode for gestational diabetes mellitus? J Soc Gynecol Investig 10: 216-221, 2003

24. Holemans K, Caluwaerts S, Poston L and Van Assche FA: Diet-induced obesity in the rat: A model for gestational diabetes mellitus. Am J Obstet Gynecol 190: 858-865, 2004.

25. Chengya L, Kristi DC and Prater MR: High-saturated-fat diet induces gestational diabetes and placental vasculopathy in C57BL/6 mice. Metabolism 59: 943-950, 2010

26. Rowan JA, Rush EC, Obolonkin V, Battin M, Wouldes T and Hague WM: Metformin in gestational diabetes: the offspring follow-up (MiG TOFU): Body composition at 2 years of age. Diabetes Care 34: 2279-2284, 2011.

27. Committee on Practice Bulletins-Obstetrics: Practice Bulletin No. 137: Gestational diabetes mellitus. Obstet Gynecol 122: 406-416, 2013.
28. Hod M, Kapur A, Sacks DA, Hadar E, Agarwal M, Di Renzo GC, Cabero Roura L, McIntyre HD, Morris JL and Divakar H: The International Federation of Gynecology and Obstetrics (FIGO) Initiative on gestational diabetes mellitus: A pragmatic guide for diagnosis, management, and care. Int J Gynaecol Obstet. 131 (Suppl 3): S173-S211, 2015.

29. Bomback AS, Rekhtman Y, Whaley-Connell AT, Kshirsagar AV, Sowers JR, Chen SC, Li S, Chinnaiyan KM, Bakris GL and McCullough PA: Gestational diabetes mellitus alone in the absence of subsequent diabetes is associated with microalbuminuria: Results from the Kidney Early Evaluation Program (KEEP). Diabetes Care 33: 2586-2591, 2010.

30. Moriya T, Matsubara M, Kishihara E, Yoshida Y and Ouchi M: Type 2 diabetic patients with diabetic retinopathy and concomitant microalbuminuria showed typical diabetic glomerulosclerosis and progressive renal dysfunction. J Diabetes Complications 30: 1111-1116, 2016.

31. American Diabetes Association: Standards of medical care in diabetes-2012. Diabetes Care 35 (Suppl 1): S11-S63, 2012.

32. Stanga Z, Nock S, Medina-Escobar P, Nydegger UE, Risch M and Risch L: Factors other than the glomerular filtration rate that determine the serum beta-2-microglobulin level. PLoS One 8: e72073, 2013

33. Gregor MF and Hotamisligil GS: Inflammatory mechanisms in obesity. Ann Rev Immunol 29: 415-445, 2011.

34. Muller S, Martin S, Koenig W, Hanifi-Moghaddam P, Rathmann W, Haastert B, Giani G, Illig T, Thorand B and Kolb H: Impaired glucose tolerance is associated with increased serum concentrations of interleukin 6 and co-regulated acute-phase proteins but not TNF-alpha or its receptors. Diabetologia 45 : 805-812, 2002.

35. Kirwan JP, Hauguel-De Mouzon S, Lepercq J, Challier JC, Huston-Presley L, Friedman JE, Kalhan SC and Catalano PM: TNF-alpha is a predictor of insulin resistance in human pregnancy. Diabetes 51: 2207-2213, 2002.

36. Cargnello $M$ and Roux PP: Activation and function of the MAPKs and their substrates, the MAPK-activated protein kinases. Microbiol Mol Biol Rev 75: 50-83, 2011.

37. Shanmugam N, Reddy MA, Guha M and Natarajan R: High glucose-induced expression of proinflammatory cytokine and chemokine genes in monocytic cells. Diabetes 52: 1256-1264, 2003.

38. Chen YY, Chen J, Hu JW, Yang ZL and Shen YL: Enhancement of lipopolysaccharide-induced toll-like receptor 2 expression and inflammatory cytokine secretion in HUVECs under high glucose conditions. Life Sci 92: 582-588, 2013

39. Kuzmicki M, Telejko B, Zonenberg A, Szamatowicz J, Kretowski A, Nikolajuk A, Laudanski P and Gorska M: Circulating pro- and anti-inflammatory cytokines in Polish women with gestational diabetes. Horm Metab Res 40: 556-560, 2008.

40. Tao L, Li D, Liu H, Jiang F, Xu Y, Cao Y, Gao R and Chen G: Neuroprotective effects of metformin on traumatic brain injury in rats associated with NF- $\kappa \mathrm{B}$ and MAPK signaling pathway. Brain Res Bull 140: 154-161, 2018.

41. Lei Y, Yi Y, Liu Y, Liu X, Keller ET, Qian CN, Zhang J and Lu Y: Metformin targets multiple signaling pathways in cancer. Chin J Cancer 36: 17, 2017.

42. Mackenzie RW and Elliott BT: Akt/PKB activation and insulin signaling: A novel insulin signaling pathway in the treatment of type 2 diabetes. Diabetes Metab Syndr Obes 7: 55-64, 2014

43. Ferreira GD, Germeyer A, de Barros Machado A, do Nascimento TL, Strowitzki T, Brum IS, von Eye Corleta H and Capp E: Metformin modulates PI3K and GLUT4 expression and $\mathrm{Akt} / \mathrm{PKB}$ phosphorylation in human endometrial stromal cells after stimulation with androgen and insulin. Eur J Obstet Gynecol Reprod Biol 175: 157-162, 2014. 